\title{
ON SINGULAR INTEGRALS ASSOCIATED TO SURFACES
}

\author{
FENG LIU
}

(Received November 20, 2012, revised April 5, 2013)

\begin{abstract}
This paper is devoted to studying the singular integral with rough kernel associated to surfaces, which contain many classical surfaces as model examples. Also, the kernel of our operator lacks smoothness on the unit sphere as well as in the radial direction. We obtain the $L^{p}$ boundedness of the singular integral under a sharp size condition on its kernels in an extrapolation argument. In addition, the corresponding results for maximal truncated singular integral operators are also established.
\end{abstract}

1. Introduction. Let $n$ be a positive integer greater than two and $S^{n-1}$ denote the unit sphere in $\boldsymbol{R}^{n}$ equipped with the normalized Lebesgue measure $d \sigma$. Let $\Omega$ be homogeneous of degree zero on $\boldsymbol{R}^{n}$ that is integrable on $S^{n-1}$ and satisfy

$$
\int_{S^{n-1}} \Omega(u) d \sigma(u)=0 .
$$

For a suitable mapping $\Gamma: \boldsymbol{R}^{n} \rightarrow \boldsymbol{R}^{d}$, we define the singular integral operators $T_{h, \Gamma}$ associated to surfaces $\left\{\Gamma(y) ; y \in \boldsymbol{R}^{n}\right\}$ by

$$
T_{h, \Gamma}(f)(x):=\text { p.v. } \int_{\boldsymbol{R}^{n}} \frac{\Omega(y) h(|y|)}{|y|^{n}} f(x-\Gamma(y)) d y .
$$

The maximal truncated singular integral operator $T_{h, \Gamma}^{*}$ is defined by

$$
T_{h, \Gamma}^{*}(f)(x):=\sup _{\varepsilon>0}\left|\int_{|y|>\varepsilon} \frac{\Omega(y) h(|y|)}{|y|^{n}} f(x-\Gamma(y)) d y\right|,
$$

where $f \in \mathscr{S}\left(\boldsymbol{R}^{d}\right)$ (the Schwartz class) and $h(\cdot) \in \Delta_{1}\left(\boldsymbol{R}^{+}\right)$. Here $\Delta_{\gamma}, \gamma>0$, is the set of all measurable functions $h$ on $\boldsymbol{R}^{+}=(0, \infty)$ satisfying

$$
\|h\|_{\Delta_{\gamma}\left(\boldsymbol{R}^{+}\right)}=\sup _{j \in \boldsymbol{Z}}\left(\int_{2^{j}}^{2^{j+1}}|h(t)|^{\gamma} \frac{d t}{t}\right)^{1 / \gamma}<\infty .
$$

One can easily check that $L^{\infty}\left(\boldsymbol{R}^{+}\right)=\Delta_{\infty}\left(\boldsymbol{R}^{+}\right) \subsetneq \Delta_{\gamma_{2}}\left(\boldsymbol{R}^{+}\right) \subsetneq \Delta_{\gamma_{1}}\left(\boldsymbol{R}^{+}\right)$for $0<\gamma_{1}<\gamma_{2}<$ $\infty$.

If $n=d, \Gamma(y)=\left(y_{1}, y_{2}, \ldots, y_{n}\right)$ and $h(t) \equiv 1$, the operator $T_{h, \Gamma}$ is the classical singular integral operator which was discussed extensively by many authors. For example, see [2] for the case $\Omega \in L \log ^{+} L\left(S^{n-1}\right)$, [1, 12] for the case $\Omega \in H^{1}\left(S^{n-1}\right)$. For $n=d$ and $\Gamma(y)=\left(y_{1}, y_{2}, \ldots, y_{n}\right)$, we denote $T_{h, \Gamma}$ by $T_{h}$. Fefferman [6] firstly introduced the case of rough radial and proved that $T_{h}$ is bounded on $L^{p}\left(\boldsymbol{R}^{n}\right)$ for $1<p<\infty$ if $\Omega$ satisfies

2010 Mathematics Subject Classification. Primary 42B20; Secondary 42B25, 42B99.

Key words and phrases. Singular integral, rough kernel, extrapolation. 
a Lipschitz condition of positive order on $S^{n-1}$ and $h \in L^{\infty}(\boldsymbol{R})$. Namazi [10] improved Fefferman's result to the case $\Omega \in L^{q}\left(S^{n-1}\right)$. Subsequently, Duoandikoetxea and Francia [4] obtained that $T_{h}$ is of type $(p, p)$ for $1<p<\infty$ provided that $\Omega \in L^{q}\left(S^{n-1}\right)$ and $h \in \Delta_{2}\left(\boldsymbol{R}^{+}\right)$. For $\Gamma(y)=\mathcal{P}(y)$, where $\mathcal{P}$ is a polynomial mapping from $\boldsymbol{R}^{n}$ to $\boldsymbol{R}^{d}$, AlSalman and Pan [3] extended the result of [4] and proved that if $\mathcal{P}(y)=-\mathcal{P}(-y)$, then $T_{h, \Gamma}$ is bounded on $L^{p}\left(\boldsymbol{R}^{d}\right)$ for $1<p<\infty$ provided that $h \in \Delta_{\gamma}\left(\boldsymbol{R}^{+}\right)$for some $\gamma>1$ and $\Omega \in L \log ^{+} L\left(S^{n-1}\right)$. In particular, Fan and Pan [8] showed that if $h \in \Delta_{\gamma}\left(\boldsymbol{R}^{+}\right)$for some $\gamma>1$ and $\Omega \in H^{1}\left(S^{n-1}\right), T_{h, \Gamma}$ is bounded on $L^{p}\left(\boldsymbol{R}^{d}\right)$ for $p$ satisfying $|1 / p-1 / 2|<$ $\min \left\{1 / 2,1 / \gamma^{\prime}\right\}$, where $1 / \gamma+1 / \gamma^{\prime}=1$. Recently, Sato [14] improved the result of [3] to the case $h \in \mathcal{N}_{1}$ or $h \in \mathcal{L}_{a}$ for some $a>2$, which are more general than $\Delta_{\gamma}\left(\boldsymbol{R}^{+}\right)$for $\gamma>1$. Here $\mathcal{L}_{\alpha}, \alpha>0$, is the set of all measurable functions $h$ on $\boldsymbol{R}^{+}$satisfying

$$
L_{\alpha}(h)=\sup _{k \in Z}\left(\int_{2^{k}}^{2^{k+1}}|h(t)|(\log (|h(t)|+2))^{\alpha} \frac{d t}{t}\right)<\infty,
$$

and $\mathcal{N}_{\alpha}, \alpha>0$, is the set of all measurable functions $h$ on $\boldsymbol{R}^{+}$satisfying $N_{\alpha}(h)=$ $\sum_{m=1} m^{\alpha} 2^{m} d_{m}(h)<\infty$ with $d_{m}(h)=\sup _{k \in \boldsymbol{Z}} 2^{-k}|E(k, m)|$, where $E(k, 1)=\{t \in$ $\left.\left(2^{k}, 2^{k+1}\right] ;|h(t)| \leq 2\right\}$ and

$$
E(k, m)=\left\{t \in\left(2^{k}, 2^{k+1}\right] ; 2^{m-1}<|h(t)| \leq 2^{m}\right\} \quad \text { for } m \geq 2 .
$$

It is easy to check that for any $\alpha>0$ and $1<\gamma<\infty$,

$$
\Delta_{\gamma}\left(\boldsymbol{R}^{+}\right) \subsetneq \mathcal{N}_{\alpha}\left(\boldsymbol{R}^{+}\right)
$$

and

$$
\mathcal{L}_{\alpha+\beta}\left(\boldsymbol{R}^{+}\right) \subsetneq \mathcal{N}_{\alpha}\left(\boldsymbol{R}^{+}\right) \subsetneq \mathcal{L}_{\alpha}\left(\boldsymbol{R}^{+}\right) \subsetneq \Delta_{1}\left(\boldsymbol{R}^{+}\right) \text {for any } \beta>1 .
$$

Now we introduce the result of [14] as follows:

Theorem A ([14]). Let $\Gamma(y)=\mathcal{P}(y)=\left(P_{1}(y), P_{2}(y), \ldots, P_{d}(y)\right)$ with $P_{j}$ being polynomials on $\boldsymbol{R}^{n}$ and $\mathcal{P}(y)=-\mathcal{P}(-y)$. Let $T_{h, \Gamma}$ and $T_{h, \Gamma}^{*}$ be given as in (1.2) and (1.3), respectively. Suppose that $\Omega$ is an element of $L \log ^{+} L\left(S^{n-1}\right)$ with satisfying (1.1) and $h \in$ $\mathcal{L}_{a}$ for some $a>2$ or $h \in \mathcal{N}_{1}$, then $T_{h, \Gamma}$ and $T_{h, \Gamma}^{*}$ are bounded on $L^{p}\left(\boldsymbol{R}^{d}\right)$ for $1<p<$ $\infty$, and the bounds are independent of the coefficients of $P_{j}$, but depend on $\operatorname{deg}\left(P_{j}\right), j=$ $1,2, \ldots, d$.

In light of the aforementioned facts concerning the above singular integrals, our main focus in this paper is to investigate the $L^{p}$ mapping properties of $T_{h, \Gamma}$ with $\Gamma(y)=P(|y|) y^{\prime}$, where $P$ is a polynomial on $\boldsymbol{R}^{+}$. More precisely, we let $\Gamma(y)=P_{N}(\varphi(|y|)) y^{\prime}$ with $P_{N}(t)=$ $\sum_{i=1}^{N} a_{i} t^{i}$ on $\boldsymbol{R}^{+}$and $\varphi$ satisfy one of the following conditions:

(i) $\varphi: \boldsymbol{R}^{+} \rightarrow(0, \infty)$ is a positive increasing $\mathcal{C}^{1}$ function such that $t \varphi^{\prime}(t) \geq C_{\varphi} \varphi(t)$ and $\varphi(2 t) \leq c_{\varphi} \varphi(t)$ for all $t>0$, where $C_{\varphi}$ and $c_{\varphi}$ are independent of $t$.

(ii) $\varphi: \boldsymbol{R}^{+} \rightarrow(0, \infty)$ is a positive decreasing $\mathcal{C}^{1}$ function such that $t \varphi^{\prime}(t) \leq-C_{\varphi} \varphi(t)$ and $\varphi(t) \leq c_{\varphi} \varphi(2 t)$ for all $t>0$, where $C_{\varphi}$ and $c_{\varphi}$ are independent of $t$.

For convenience, we denote by $\mathfrak{F}_{1}$ (or $\mathfrak{F}_{2}$ ) the set of all functions which satisfy the condition (i) (or (ii)). 
REMARK 1.1. There are some model examples in the class $\mathfrak{F}_{1}$, such as $t^{\alpha}(\alpha>0)$, $t^{\alpha}(\ln (1+t))^{\beta}(\alpha, \beta>0), t \ln \ln (e+t)$, real-valued polynomials $P$ on $\boldsymbol{R}$ with positive coefficients and $P(0)=0$ and so on. We now give examples in the class $\mathfrak{F}_{2}$ such as $t^{\delta}(\delta<0)$ and $t^{-1} \ln (1+1 / t)$. It should be pointed out that there are two important facts as follows:

(a) If $\varphi(t) \in \mathcal{C}^{1}\left(\boldsymbol{R}^{+}\right)$is nonnegative and increasing (resp. decreasing) on $\boldsymbol{R}^{+}$and $\varphi(t) /\left(t \varphi^{\prime}(t)\right)$ is bounded on $\boldsymbol{R}^{+}$, then $\lim _{t \rightarrow 0} \varphi(t)=0\left(\right.$ resp. $\left.\lim _{t \rightarrow 0} \varphi(t)=+\infty\right)$ and $\lim _{t \rightarrow+\infty} \varphi(t)=+\infty$ (resp. $\left.\lim _{t \rightarrow+\infty} \varphi(t)=0\right)$ (see [5]).

(b) For $\varphi \in \mathfrak{F}_{1}\left(\right.$ or $\mathfrak{F}_{2}$ ), there exists a constant $B_{\varphi}>1$ such that $\varphi(2 t) \geq B_{\varphi} \varphi(t)$ (or $\left.\varphi(t) \geq B_{\varphi} \varphi(2 t)\right)$ (see [9]).

Our main results can be formulated as follows:

THEOREM 1.2. Let $n=d$ and $\Gamma(y)=P_{N}(\varphi(|y|)) y^{\prime}$ with $P_{N}(t)=\sum_{i=1}^{N} a_{i} t^{i}$ and $\varphi \in \mathfrak{F}_{1}$ or $\mathfrak{F}_{2}$. Let $T_{h, \Gamma}$ be given as in (1.2). Suppose that $\Omega \in L^{q}\left(S^{n-1}\right)$ with satisfying (1.1) and $h \in \Delta_{\gamma}\left(\boldsymbol{R}^{+}\right)$for some $q, \gamma \in(1,2]$. Then

$$
\left\|T_{h, \Gamma}(f)\right\|_{L^{p}\left(\boldsymbol{R}^{n}\right)} \leq C_{p}(q-1)^{-1}(\gamma-1)^{-1}\|\Omega\|_{L^{q}\left(S^{n-1}\right)}\|h\|_{\Delta_{\gamma}\left(\boldsymbol{R}^{+}\right)}\|f\|_{L^{p}\left(\boldsymbol{R}^{n}\right)}
$$

for $1<p<\infty$, where the constant $C_{p}$ is independent of $q, \gamma, \Omega, h$ and the coefficients of $P_{N}$, but depends on $\varphi$ and $N$.

THEOREM 1.3. Let $d, \Gamma, P_{N}, \varphi, \Omega, h$ be as in Theorem 1.2 and $T_{h, \Gamma}^{*}$ be given as in (1.3). Then

$$
\left\|T_{h, \Gamma}^{*}(f)\right\|_{L^{p}\left(\boldsymbol{R}^{n}\right)} \leq C_{p}(q-1)^{-1}(\gamma-1)^{-1}\|\Omega\|_{L^{q}\left(S^{n-1}\right)}\|h\|_{\Delta_{\gamma}\left(\boldsymbol{R}^{+}\right)}\|f\|_{L^{p}\left(\boldsymbol{R}^{n}\right)}
$$

for $1<p<\infty$, where the constant $C_{p}$ is independent of $q, \gamma, \Omega, h$ and the coefficients of $P_{N}$, but depends on $\varphi$ and $N$.

TheOREM 1.4. Let $d, \Gamma, P_{N}, \varphi$ be as in Theorem 1.2. Let $T_{h, \Gamma}$ and $T_{h, \Gamma}^{*}$ be given as in (1.2) and (1.3), respectively. Suppose that $\Omega \in L \log ^{+} L\left(S^{n-1}\right)$ with satisfying (1.1) and $h \in \mathcal{L}_{a}$ for some $a>2$ or $h \in \mathcal{N}_{1}$, then $T_{h, \Gamma}$ and $T_{h, \Gamma}^{*}$ are bounded on $L^{p}\left(\boldsymbol{R}^{n}\right)$ for $1<p<\infty$, and the bounds are independent of the coefficients of $P_{N}$, but depend on $\varphi$ and $N$.

As several applications of Theorem 1.4, we have the following corollaries.

Corollary 1.5. Let $n=d$ and $\Gamma(y)=P_{N}(|y|) y^{\prime}$ with $P_{N}(t)=\sum_{i=1}^{N} a_{i} t^{i}$. Let $T_{h, \Gamma}$ and $T_{h, \Gamma}^{*}$ be given as in (1.2) and (1.3), respectively. Suppose that $\Omega \in L \log ^{+} L\left(S^{n-1}\right)$ with satisfying (1.1) and $h \in \mathcal{L}_{a}$ for some $a>2$ or $h \in \mathcal{N}_{1}$, then $T_{h, \Gamma}$ and $T_{h, \Gamma}^{*}$ are bounded on $L^{p}\left(\boldsymbol{R}^{n}\right)$ for $1<p<\infty$, and the bounds are independent of the coefficients of $P_{N}$, but depend on $N$.

COROLlaRY 1.6. Let $n=d$ and $\Gamma(y)=\varphi(|y|) y^{\prime}$ with $\varphi \in \mathfrak{F}_{1}$ or $\mathfrak{F}_{2}$. Let $T_{h, \Gamma}$ and $T_{h, \Gamma}^{*}$ be given as in (1.2) and (1.3), respectively. Suppose that $\Omega \in L \log ^{+} L\left(S^{n-1}\right)$ with satisfying (1.1) and $h \in \mathcal{L}_{a}$ for some $a>2$ or $h \in \mathcal{N}_{1}$, then $T_{h, \Gamma}$ and $T_{h, \Gamma}^{*}$ are bounded on $L^{p}\left(\boldsymbol{R}^{n}\right)$ for $1<p<\infty$, and the bounds depend on $\varphi$. 
This paper is organized as follows. In Section 2 we shall establish some preliminary lemmas. The proofs of main results will be given in Section 3. We remark that the proof of Theorem 1.2 is based on the method of [14]. Employing the idea in [7], we shall prove Theorem 1.3. The proof of Theorem 1.4 is based on an extrapolation method which was originally introduced by Yano (see [16]) and developed by Sato (see [13, 14]).

Throughout the rest of the paper, the letter $C$ will stand for a positive constant not necessarily the same one at each occurrence but is independent of the essential variables. Also, we let $x^{\prime}=x /|x|$ for $x \in \boldsymbol{R}^{n} \backslash\{0\}$ and $p^{\prime}$ denote the conjugate index of $p$, that is, $1 / p+1 / p^{\prime}=1$.

2. Preliminary lemmas. Let us begin by introducing some notations and establishing some lemmas. We denote $P_{\lambda}(t)=\sum_{i=1}^{\lambda} a_{i} t^{i}$ for $\lambda \in\{1,2, \ldots, N\}$ and $P_{0}(t)=0$.

LEMMA 2.1 ([11, p. 186, Corollary]). Suppose that $\Phi(t)=t^{\alpha_{1}}+\mu_{2} t^{\alpha_{2}}+\cdots+$ $\mu_{n} t^{\alpha_{n}}$ and $\Psi \in C^{1}[a, b]$, where $\mu_{2}, \ldots, \mu_{n}$ are real parameters, and $\alpha_{1}, \ldots, \alpha_{n}$ are distinct positive (not necessarily integer) exponents. Then

$$
\left|\int_{a}^{b} \exp (i \lambda \Phi(t)) \Psi(t) d t\right| \leq C \lambda^{-\varepsilon}\left\{\sup _{a \leq t \leq b}|\Psi(t)|+\int_{a}^{b}\left|\Psi^{\prime}(t)\right| d t\right\},
$$

with $\varepsilon=\min \left\{1 / \alpha_{1}, 1 / n\right\}$ and $C$ does not depend on $\mu_{2}, \ldots, \mu_{n}$ as long as $0 \leq a<b \leq 1$.

Applying Lemma 2.1, we have the following results, which will play a key role in the estimates about Fourier transforms of some measures on $\boldsymbol{R}^{n}$.

LEMMA 2.2. Let $\Omega \in L^{q}\left(S^{n-1}\right)$ for some $q>1$ and $P_{\lambda}$ be as above. If $\varphi \in \mathfrak{F}_{1}$, then for any $0<\varepsilon<\min \left\{1 / q^{\prime}, 1 / \lambda\right\}$, we have

$$
\int_{r / 2}^{r}\left|\int_{S^{n-1}} \Omega\left(u^{\prime}\right) e^{-i P_{\lambda}(\varphi(t)) \xi \cdot u^{\prime}} d \sigma\left(u^{\prime}\right)\right|^{2} \frac{d t}{t} \leq C(\varphi)\|\Omega\|_{L^{q}\left(S^{n-1}\right)}^{2}\left|\varphi(r)^{\lambda} a_{\lambda} \xi\right|^{-\varepsilon}
$$

for $\lambda \in\{1,2, \ldots, N\}$ and any $r>0$. The constant $C(\varphi)$ is independent of $\Omega, q$ and the coefficients of $P_{\lambda}$, but depends on $\varphi$.

Proof. By the change of the variables, we have

$$
\begin{aligned}
\int_{r / 2}^{r} \mid \int_{S^{n-1}} & \left.\Omega\left(u^{\prime}\right) e^{-i P_{\lambda}(\varphi(t)) \xi \cdot u^{\prime}} d \sigma\left(u^{\prime}\right)\right|^{2} \frac{d t}{t} \\
& =\int_{\varphi(r / 2)}^{\varphi(r)}\left|\int_{S^{n-1}} \Omega\left(u^{\prime}\right) e^{-i P_{\lambda}(t) \xi \cdot u^{\prime}} d \sigma\left(u^{\prime}\right)\right|^{2} \frac{d t}{\varphi^{-1}(t) \varphi^{\prime}\left(\varphi^{-1}(t)\right)} \\
& \leq \frac{1}{C_{\varphi}} \int_{\varphi(r / 2)}^{\varphi(r)}\left|\int_{S^{n-1}} \Omega\left(u^{\prime}\right) e^{-i P_{\lambda}(t) \xi \cdot u^{\prime}} d \sigma\left(u^{\prime}\right)\right|^{2} \frac{d t}{t} \\
& \leq \frac{1}{C_{\varphi} \varphi(r / 2)} \int_{\varphi(r / 2)}^{\varphi(r)}\left|\int_{S^{n-1}} \Omega\left(u^{\prime}\right) e^{-i P_{\lambda}(t) \xi \cdot u^{\prime}} d \sigma\left(u^{\prime}\right)\right|^{2} d t \\
& \leq \frac{c_{\varphi}}{C_{\varphi}} \int_{\zeta}^{1}\left|\int_{S^{n-1}} \Omega\left(u^{\prime}\right) e^{-i P_{\lambda}(\varphi(r) t) \xi \cdot u^{\prime}} d \sigma\left(u^{\prime}\right)\right|^{2} d t
\end{aligned}
$$


where $\varsigma=\varphi(r / 2) / \varphi(r) \in\left[1 / c_{\varphi}, 1\right)$. By Lemma 2.1 and Hölder inequality, we have

$$
\begin{aligned}
\int_{\zeta}^{1} \mid \int_{S^{n-1}} & \left.\Omega\left(u^{\prime}\right) e^{-i P_{\lambda}(\varphi(r) t) \xi \cdot u^{\prime}} d \sigma\left(u^{\prime}\right)\right|^{2} d t \\
& =\int_{\zeta}^{1} \iint_{\left(S^{n-1}\right)^{2}} \Omega\left(u^{\prime}\right) \overline{\Omega(\theta)} e^{-i P_{\lambda}(\varphi(r) t) \xi \cdot\left(u^{\prime}-\theta\right)} d \sigma\left(u^{\prime}\right) d \sigma(\theta) d t \\
& \leq \iint_{\left(S^{n-1}\right)^{2}}\left|\Omega\left(u^{\prime}\right) \overline{\Omega(\theta)}\right| \int_{\zeta}^{1} e^{-i P_{\lambda}(\varphi(r) t) \xi \cdot\left(u^{\prime}-\theta\right)} d t \mid d \sigma\left(u^{\prime}\right) d \sigma(\theta) \\
& \leq \iint_{\left(S^{n-1}\right)^{2}}\left|\Omega\left(u^{\prime}\right) \overline{\Omega(\theta)}\right| \min \left\{\log c_{\varphi},\left|\varphi(r)^{\lambda} a_{\lambda} \xi \cdot\left(u^{\prime}-\theta\right)\right|^{-1 / \lambda}\right\} d \sigma\left(u^{\prime}\right) d \sigma(\theta) \\
& \leq C_{\varepsilon}\left(\log c_{\varphi}\right)^{1-\varepsilon}\|\Omega\|_{L^{q}\left(S^{n-1}\right)}^{2}\left|\varphi(r)^{\lambda} a_{\lambda} \xi\right|^{-\varepsilon},
\end{aligned}
$$

which combining with (2.1) yields Lemma 2.2.

By an argument similar to that of Lemma 2.2, we obtain

LEMMA 2.3. Let $\Omega \in L^{q}\left(S^{n-1}\right)$ for some $q>1$ and $P_{\lambda}$ be as above. If $\varphi \in \mathfrak{F}_{2}$, then for any $0<\varepsilon<\min \left\{1 / q^{\prime}, 1 / \lambda\right\}$, we have

$$
\int_{r / 2}^{r}\left|\int_{S^{n-1}} \Omega\left(u^{\prime}\right) e^{-i P_{\lambda}(\varphi(t)) \xi \cdot u^{\prime}} d \sigma\left(u^{\prime}\right)\right|^{2} \frac{d t}{t} \leq C(\varphi)\|\Omega\|_{L^{q}\left(S^{n-1}\right)}^{2}\left|\varphi(r / 2)^{\lambda} a_{\lambda} \xi\right|^{-\varepsilon}
$$

for $\lambda \in\{1,2, \ldots, N\}$ and any $r>0$. The constant $C(\varphi)$ is independent of $\Omega$ and the coefficients of $P_{\lambda}$, but depends on $\varphi$.

3. Proofs of main results. Let $\Gamma$ be as in Theorem 1.2 and $P_{\lambda}$ as in Section 2. Let $\beta \geq 2$ and $E_{k}=\left\{u \in \boldsymbol{R}^{n} ; \beta^{k}<|u| \leq \beta^{k+1}\right\}$. Define the measures $\left\{\sigma_{k, \lambda}\right\}_{k \in \boldsymbol{Z}}$ and $\left\{\left|\sigma_{k, \lambda}\right|\right\}_{k \in \boldsymbol{Z}}$ by

$$
\begin{aligned}
& \int_{\boldsymbol{R}^{n}} f(x) d \sigma_{k, \lambda}(x)=\int_{E_{k}} \frac{\Omega(u) h(|u|)}{|u|^{n}} f\left(P_{\lambda}(\varphi(|u|)) u^{\prime}\right) d u, \\
& \int_{\boldsymbol{R}^{n}} f(x) d\left|\sigma_{k, \lambda}\right|(x)=\int_{E_{k}} \frac{|\Omega(u) h(|u|)|}{|u|^{n}} f\left(P_{\lambda}(\varphi(|u|)) u^{\prime}\right) d u
\end{aligned}
$$

for $\lambda \in\{0,1, \ldots, N\}$. It is easy to see that

$$
T_{h, \Gamma}(f)(x)=\sum_{k \in \boldsymbol{Z}} \sigma_{k, N} * f(x) .
$$

LEMmA 3.1. Let $\Omega \in L^{q}\left(S^{n-1}\right)$ with satisfying (1.1) and $h \in \Delta_{\gamma}\left(\boldsymbol{R}^{+}\right)$for some $q, \gamma \in(1,2]$. Suppose $\varphi \in \mathfrak{F}_{1}$ or $\mathfrak{F}_{2}$. For any $\lambda \in\{1,2, \ldots, N\}$, there exists a constant $C>0$ such that

(i)

$$
\left\|\sigma_{k, \lambda}\right\| \leq C \log \beta\|\Omega\|_{L^{q}\left(S^{n-1}\right)}\|h\|_{\Delta_{\gamma}\left(\boldsymbol{R}^{+}\right)},
$$

(ii) if $\varphi \in \mathfrak{F}_{1}$, then

$$
\left|\widehat{\sigma_{k, \lambda}}(\xi)-\widehat{\sigma_{k, \lambda-1}}(\xi)\right| \leq C \log \beta\|\Omega\|_{L^{q}\left(S^{n-1}\right)}\|h\|_{\Delta_{\gamma}\left(\boldsymbol{R}^{+}\right)}\left|\varphi\left(\beta^{k+1}\right)^{\lambda} a_{\lambda} \xi\right|^{1 /\left(\lambda q^{\prime} \gamma^{\prime}\right)},
$$




$$
\left|\widehat{\sigma_{k, \lambda}}(\xi)\right| \leq C \log \beta\|\Omega\|_{L^{q}\left(S^{n-1}\right)}\|h\|_{\Delta_{\gamma}\left(\boldsymbol{R}^{+}\right)}\left|\varphi\left(\beta^{k}\right)^{\lambda} a_{\lambda} \xi\right|^{-1 /\left(2 \lambda q^{\prime} \gamma^{\prime}\right)},
$$

(iii) if $\varphi \in \mathfrak{F}_{2}$, then

$$
\begin{gathered}
\left|\widehat{\sigma_{k, \lambda}}(\xi)-\widehat{\sigma_{k, \lambda-1}}(\xi)\right| \leq C \log \beta\|\Omega\|_{L^{q}\left(S^{n-1}\right)}\|h\|_{\Delta_{\gamma}\left(\boldsymbol{R}^{+}\right)}\left|\varphi\left(\beta^{k}\right)^{\lambda} a_{\lambda} \xi\right|^{1 /\left(\lambda q^{\prime} \gamma^{\prime}\right)}, \\
\left|\widehat{\sigma_{k, \lambda}}(\xi)\right| \leq C \log \beta\|\Omega\|_{L^{q}\left(S^{n-1}\right)}\|h\|_{\Delta_{\gamma}\left(\boldsymbol{R}^{+}\right)}\left|\varphi\left(\beta^{k+1}\right)^{\lambda} a_{\lambda} \xi\right|^{-1 /\left(2 \lambda q^{\prime} \gamma^{\prime}\right)} .
\end{gathered}
$$

The constant $C$ is independent of $\beta, q, \gamma$ and the coefficients of $P_{N}$, but depends on $\varphi$.

PROOF. (3.2) is obvious. We only prove (ii) since we can obtain (iii) similarly. Since $\varphi$ is increasing, then

$$
\begin{aligned}
\left|\widehat{\sigma_{k, \lambda}}(\xi)-\widehat{\sigma_{k, \lambda-1}}(\xi)\right| & \leq \int_{E_{k}} \frac{|\Omega(u) h(|u|)|}{|u|^{n}}\left|e^{-2 \pi i P_{\lambda}(\varphi(|u|)) u^{\prime} \cdot \xi}-e^{-2 \pi i P_{\lambda-1}(\varphi(|u|)) u^{\prime} \cdot \xi}\right| d u \\
& \leq C \min \left\{1,\left|\varphi\left(\beta^{k+1}\right)^{\lambda} a_{\lambda} \xi\right|\right\} \int_{\beta^{k}}^{\beta^{k+1}}|h(t)| \frac{d t}{t}\|\Omega\|_{L^{1}\left(S^{n-1}\right)} \\
& \leq C \log \beta\|\Omega\|_{L^{q}\left(S^{n-1}\right)}\|h\|_{\Delta_{\gamma}\left(\boldsymbol{R}^{+}\right)}\left(\left|\varphi\left(\beta^{k+1}\right)^{\lambda} a_{\lambda} \xi\right|\right)^{1 /\left(\lambda q^{\prime} \gamma^{\prime}\right)} .
\end{aligned}
$$

Thus (3.3) is proved. We now prove (3.4). By the change of the variables and the Hölder inequality, we have

(3.7)

$$
\begin{aligned}
& \left|\widehat{\sigma_{k, \lambda}}(\xi)\right| \\
& =\left|\int_{\beta^{k}}^{\beta^{k+1}} \int_{S^{n-1}} \Omega\left(u^{\prime}\right) e^{-2 \pi i P_{\lambda}(\varphi(t)) u^{\prime} \cdot \xi} d \sigma\left(u^{\prime}\right) h(t) \frac{d t}{t}\right| \\
& \leq(\log \beta)^{1 / \gamma}\|h\|_{\Delta_{\gamma}\left(\boldsymbol{R}^{+}\right)}\left(\int_{\beta^{k}}^{\beta^{k+1}}\left|\int_{S^{n-1}} \Omega\left(u^{\prime}\right) e^{-2 \pi i P_{\lambda}(\varphi(t)) u^{\prime} \cdot \xi} d \sigma\left(u^{\prime}\right)\right|^{\gamma^{\prime}} \frac{d t}{t}\right)^{1 / \gamma^{\prime}}
\end{aligned}
$$

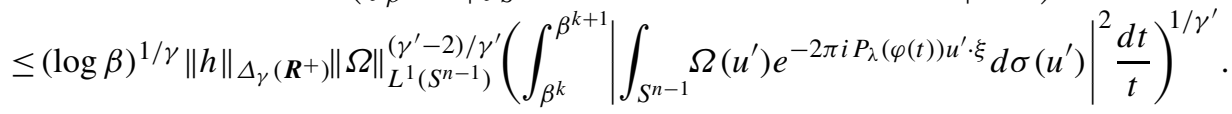

For any $\beta \geq 2$, choosing $v \in Z$ such that $2^{v}<\beta \leq 2^{v+1}$, we have from Lemma 2.2 that

$$
\begin{aligned}
\int_{\beta^{k}}^{\beta^{k+1}} & \left|\int_{S^{n-1}} \Omega\left(u^{\prime}\right) e^{-2 \pi i P_{\lambda}(\varphi(t)) u^{\prime} \cdot \xi} d \sigma\left(u^{\prime}\right)\right|^{2} \frac{d t}{t} \\
& \leq \sum_{j=0}^{v} \int_{\beta^{k} 2^{j}}^{\beta^{k} 2^{j+1}}\left|\int_{S^{n-1}} \Omega\left(u^{\prime}\right) e^{-2 \pi i P_{\lambda}(\varphi(t)) u^{\prime} \cdot \xi} d \sigma\left(u^{\prime}\right)\right|^{2} \frac{d t}{t} \\
& \leq \sum_{j=0}^{v} C(\varphi)\|\Omega\|_{L^{q}\left(S^{n-1}\right)}^{2}\left|\varphi\left(\beta^{k} 2^{j+1}\right)^{\lambda} a_{\lambda} \xi\right|^{-\varepsilon} \\
& \leq C(\varphi) \log \beta\|\Omega\|_{L^{q}\left(S^{n-1}\right)}\|h\|_{\Delta_{\gamma}\left(\boldsymbol{R}_{+}\right)}\left|\varphi\left(\beta^{k}\right)^{\lambda} a_{\lambda} \xi\right|^{-\varepsilon}
\end{aligned}
$$

for $0<\varepsilon<\min \left\{1 / q^{\prime}, 1 / \lambda\right\}$. (3.4) follows from this inequality with taking $\varepsilon=1 /\left(2 q^{\prime} \lambda\right)$ and (3.7). Lemma 3.1 is completed. 
Let $\psi \in \mathcal{C}_{0}^{\infty}(\boldsymbol{R})$ be supported in $\{|t| \leq 1\}$ and $\psi(t) \equiv 1$ for $|t|<1 / 2$. Define the measures $\left\{\mu_{k, \lambda}^{i}\right\}_{k \in \boldsymbol{Z}}(i=1,2)$ on $\boldsymbol{R}^{n}$ by

$$
\begin{gathered}
\widehat{\mu_{k, \lambda}^{1}}(\xi)=\widehat{\sigma_{k, \lambda}}(\xi) \prod_{j=\lambda+1}^{N} \psi\left(\left|\varphi\left(\beta^{k}\right)^{j} a_{j} \xi\right|\right)-\widehat{\sigma_{k, \lambda-1}}(\xi) \prod_{j=\lambda}^{N} \psi\left(\left|\varphi\left(\beta^{k}\right)^{j} a_{j} \xi\right|\right), \\
\widehat{\mu_{k, \lambda}^{2}}(\xi)=\widehat{\sigma_{k, \lambda}}(\xi) \prod_{j=\lambda+1}^{N} \psi\left(\left|\varphi\left(\beta^{k+1}\right)^{j} a_{j} \xi\right|\right)-\widehat{\sigma_{k, \lambda-1}}(\xi) \prod_{j=\lambda}^{N} \psi\left(\left|\varphi\left(\beta^{k+1}\right)^{j} a_{j} \xi\right|\right)
\end{gathered}
$$

for $k \in Z$ and $\lambda \in\{1,2, \ldots, N\}$. It is easy to see that

$$
\sigma_{k, N}=\sum_{\lambda=1}^{N} \mu_{k, \lambda}^{i}, \text { if } \varphi \in \mathfrak{F}_{i}
$$

for $i=1,2$. Here we use the convention $\Pi_{j \in \emptyset} a_{j}=1$. By the definition of $\mu_{k, \lambda}^{i}(i=1,2)$, Lemma 3.1, the change of the variables and a well-known result on maximal functions (see [8]), we have

(i) if $\varphi \in \mathfrak{F}_{1}$, then

$$
\begin{aligned}
& \widehat{\mid \mu_{k, \lambda}^{1}}(\xi) \mid \leq C \log \beta\|\Omega\|_{L^{q}\left(S^{n-1}\right)}\|h\|_{\Delta_{\gamma}\left(\boldsymbol{R}^{+}\right)} \\
& \quad \times\left(\min \left\{1,\left|\varphi\left(\beta^{k+1}\right)^{\lambda} a_{\lambda} \xi\right|,\left|\varphi\left(\beta^{k}\right)^{\lambda} a_{\lambda} \xi\right|^{-1}\right\}\right)^{1 /\left(\lambda q^{\prime} \gamma^{\prime}\right)},
\end{aligned}
$$

(ii) if $\varphi \in \mathfrak{F}_{2}$, then

$$
\begin{aligned}
& \widehat{\mid \mu_{k, \lambda}^{2}}(\xi) \mid \leq C \log \beta\|\Omega\|_{L^{q}\left(S^{n-1}\right)}\|h\|_{\Delta_{\gamma}\left(\boldsymbol{R}^{+}\right)} \\
& \quad \times\left(\min \left\{1,\left|\varphi\left(\beta^{k}\right)^{\lambda} a_{\lambda} \xi\right|,\left|\varphi\left(\beta^{k+1}\right)^{\lambda} a_{\lambda} \xi\right|^{-1}\right\}\right)^{1 /\left(\lambda q^{\prime} \gamma^{\prime}\right)},
\end{aligned}
$$

and

$$
\mu_{\lambda}^{*, i}(f)(\xi) \leq C \mathcal{M}\left(\sigma_{\lambda}^{*}(|f|)\right)(\xi)+C \mathcal{M}\left(\sigma_{\lambda-1}^{*}(|f|)\right)(\xi)
$$

for $i=1,2$ and $\lambda \in\{1,2, \ldots, N\}$, where $\mathcal{M}$ is the standard Hardy-Littlewood maximal operator and

$$
\begin{gathered}
\sigma_{\lambda}^{*}(f)(\xi)=\sup _{k \in \boldsymbol{Z}}|| \sigma_{k, \lambda}|* f(\xi)|, \\
\mu_{\lambda}^{*, i}(f)(\xi)=\sup _{k \in \boldsymbol{Z}}|| \mu_{k, \lambda}^{i}|* f(\xi)|, \quad \text { for } i=1,2 .
\end{gathered}
$$

In what follows, we set $\beta=2^{q^{\prime} \gamma^{\prime}}$ and $A=q^{\prime} \gamma^{\prime}\|\Omega\|_{L^{q}\left(S^{n-1}\right)}\|h\|_{\Delta_{\gamma}\left(\boldsymbol{R}^{+}\right)}$. Then, we have the following result.

LEMmA 3.2. Let $\varphi \in \mathfrak{F}_{1}$ or $\mathfrak{F}_{2}$. For $\lambda \in\{0,1, \ldots, N\}$, the operator $\sigma_{\lambda}^{*}$ satisfies

$$
\left\|\sigma_{\lambda}^{*}(f)\right\|_{L^{p}\left(\boldsymbol{R}^{n}\right)} \leq C_{p} A\|f\|_{L^{p}\left(\boldsymbol{R}^{n}\right)}
$$

for all $1<p<\infty$. The constant $C_{p}$ is independent of $q, \gamma$ and the coefficients of $P_{\lambda}$, but depends on $\varphi$. 
Proof. We prove the estimate (3.12) provided that $\varphi \in \mathfrak{F}_{1}$. The other case can be proved similarly. We prove this lemma by induction on $\lambda$.

CASE 1. It is easy to check that $\sigma_{0}^{*}(f)(\xi) \leq C A|f(\xi)|$, which implies the estimate (3.12) for $\lambda=0$.

CASE 2. Let $m \in\{1,2, \ldots, N\}$ and suppose that (3.12) holds for $\lambda=m-1$. We will prove (3.12) for $\lambda=m$. Let $\psi \in \mathcal{C}_{0}^{\infty}(\boldsymbol{R})$ be as above. Define the Borel measures $\left\{\omega_{k, \lambda}\right\}_{k \in \boldsymbol{Z}}$ on $\boldsymbol{R}^{n}$ by

$$
\widehat{\omega_{k, \lambda}}(\xi)=\widehat{\left|\sigma_{k, \lambda}\right|}(\xi)-\psi\left(\left|\varphi\left(2^{q^{\prime} \gamma^{\prime} k}\right)^{\lambda} a_{\lambda} \xi\right|\right)\left|\widehat{\sigma_{k, \lambda-1}}\right|(\xi)
$$

for $\xi \in \boldsymbol{R}^{n}$ and $\lambda \in\{1,2, \ldots, N\}$. By the proof of Lemma 3.1, it is easy to obtain that

$$
\left|\widehat{\omega_{k, \lambda}}(\xi)\right| \leq C A\left(\min \left\{1,\left|\varphi\left(2^{q^{\prime} \gamma^{\prime}(k+1)}\right)^{\lambda} a_{\lambda} \xi\right|,\left|\varphi\left(2^{q^{\prime} \gamma^{\prime} k}\right)^{\lambda} a_{\lambda} \xi\right|^{-1}\right\}\right)^{1 /\left(\lambda q^{\prime} \gamma^{\prime}\right)}
$$

and

$$
\begin{aligned}
& \sigma_{m}^{*}(f)(\xi) \leq G_{m}(f)(\xi)+C \mathcal{M}\left(\sigma_{m-1}^{*}(f)\right)(\xi), \\
& \omega_{m}^{*}(f)(\xi) \leq G_{m}(f)(\xi)+C \mathcal{M}\left(\sigma_{m-1}^{*}(f)\right)(\xi),
\end{aligned}
$$

where

$$
\omega_{\lambda}^{*}(f)(\xi)=\sup _{k \in \boldsymbol{Z}}|| \omega_{k, \lambda}|* f(\xi)| \quad \text { and } \quad G_{\lambda}(f)(\xi)=\left(\sum_{k \in \boldsymbol{Z}}\left|\omega_{k, \lambda} * f(\xi)\right|^{2}\right)^{1 / 2}
$$

for $\lambda \in\{1,2, \ldots, N\}$. It follows from our assumption and the $L^{p}$ mapping properties of $\mathcal{M}$ that

$$
\left\|\mathcal{M}\left(\sigma_{m-1}^{*}(f)\right)\right\|_{L^{p}\left(\boldsymbol{R}^{n}\right)} \leq C_{p}\left\|\sigma_{m-1}^{*}(f)\right\|_{L^{p}\left(\boldsymbol{R}^{n}\right)} \leq C_{p} A\|f\|_{L^{p}\left(\boldsymbol{R}^{n}\right)}
$$

for $1<p<\infty$, where the constant $C_{p}$ is independent of $q, \gamma$ and the coefficients of $P_{N}$, but depends on $\varphi$. By (3.14), it suffices to prove that

$$
\left\|G_{m}(f)\right\|_{L^{p}\left(\boldsymbol{R}^{n}\right)} \leq C_{p} A\|f\|_{L^{p}\left(\boldsymbol{R}^{n}\right)}
$$

for all $p \in(1, \infty)$ and the constant $C_{p}$ is as above. By the well-known property of Rademacher's functions, (3.17) follows from the following lemma.

LEMMA 3.3. Let $V_{\varepsilon}^{m}(f)(\xi)=\sum_{k \in \boldsymbol{Z}} \varepsilon_{k} \omega_{k, m} * f(\xi)$ with $\varepsilon=\left\{\varepsilon_{k}\right\}, \varepsilon_{k}=1$ or -1 . Then

$$
\left\|V_{\varepsilon}^{m}(f)\right\|_{L^{p}\left(\boldsymbol{R}^{n}\right)} \leq C_{p} A\|f\|_{L^{p}\left(\boldsymbol{R}^{n}\right)}
$$

for $1<p<\infty$. The constant $C_{p}$ is independent of $q, \gamma$ and the coefficients of $P_{N}$, but depends on $\varphi$.

Proof of Lemma 3.3. Choose a sequence of nonnegative functions $\left\{\Psi_{k}\right\}_{k \in \boldsymbol{Z}}$ in $\mathcal{C}_{0}^{\infty}(\boldsymbol{R})$ such that

$$
\begin{gathered}
\operatorname{supp}\left(\Psi_{k}\right) \subset\left[\varphi\left(2^{q^{\prime} \gamma^{\prime}(k+1)}\right)^{-\lambda}, \varphi\left(2^{q^{\prime} \gamma^{\prime}(k-1)}\right)^{-\lambda}\right], \quad \sum_{k \in \boldsymbol{Z}} \Psi_{k}^{2}(t)=1, \\
\left|(d / d t)^{j} \Psi_{k}(t)\right| \leq C_{j}|t|^{-j}(j=1,2, \ldots) \quad \text { for all } t>0 \text { and } j \in N,
\end{gathered}
$$


where $C_{j}$ are independent of $q, \gamma, k$. Define the Fourier multiplier operator $S_{j}$ by

$$
\widehat{S_{j}(f)}(\xi)=\Psi_{j}\left(\left|a_{\lambda} \xi\right|\right) \hat{f}(\xi), \quad \text { for } j \in \boldsymbol{Z} \text {. }
$$

Then

$$
\begin{aligned}
V_{\varepsilon}^{m}(f)(\xi) & =\sum_{k \in \boldsymbol{Z}} \varepsilon_{k} \omega_{k, m} * f(\xi) \\
& =\sum_{k \in \boldsymbol{Z}} \varepsilon_{k} \omega_{k, m} * \sum_{k \in \boldsymbol{Z}} S_{j+k} S_{j+k} f(\xi) \\
& =\sum_{j \in \boldsymbol{Z}} \sum_{k \in \boldsymbol{Z}} \varepsilon_{k} S_{j+k}\left(\omega_{k, m} * S_{j+k} f\right)(\xi) \\
& :=\sum_{j \in \boldsymbol{Z}} V_{j}^{m}(f)(\xi)
\end{aligned}
$$

By the Littlewood-Paley theory, we have

$$
\left\|V_{j}^{m}(f)\right\|_{L^{p}\left(\boldsymbol{R}^{n}\right)} \leq C_{p}\left\|\left(\sum_{k \in \boldsymbol{Z}}\left|\omega_{k, m} * S_{j+k} f(\cdot)\right|^{2}\right)^{1 / 2}\right\|_{L^{p}\left(\boldsymbol{R}^{n}\right)}
$$

for $1<p<\infty$. This combining with the Plancherel theorem yields

$$
\begin{aligned}
\left\|V_{j}^{m}(f)\right\|_{L^{2}\left(\boldsymbol{R}^{n}\right)}^{2} & \leq C\left\|\left(\sum_{k \in \boldsymbol{Z}}\left|\omega_{k, m} * S_{j+k} f(\cdot)\right|^{2}\right)^{1 / 2}\right\|_{L^{2}\left(\boldsymbol{R}^{n}\right)}^{2} \\
& \leq C \sum_{k \in \boldsymbol{Z}} \int_{D_{j+k}}\left|\widehat{\omega_{k, m}}(\xi)\right|^{2}|\hat{f}(\xi)|^{2} d \xi,
\end{aligned}
$$

where

$$
D_{k}=\left\{\xi \in \boldsymbol{R}^{n} ; \varphi\left(2^{q^{\prime} \gamma^{\prime}(k+1)}\right)^{-\lambda} \leq\left|a_{\lambda} \xi\right| \leq \varphi\left(2^{q^{\prime} \gamma^{\prime}(k-1)}\right)^{-\lambda}\right\} .
$$

By (3.13), we get

$$
\left\|V_{j}^{m}(f)\right\|_{L^{2}\left(\boldsymbol{R}^{n}\right)} \leq C A\left(B_{\varphi}^{-j+2} \chi_{\{j \geq 2\}}+B_{\varphi}^{j+1} \chi_{\{j<2\}}\right)\|f\|_{L^{2}\left(\boldsymbol{R}^{n}\right)} .
$$

This together with (3.18) implies

$$
\left\|V_{\varepsilon}^{m}(f)\right\|_{L^{2}\left(\boldsymbol{R}^{n}\right)} \leq C(\varphi) A\|f\|_{L^{2}\left(\boldsymbol{R}^{n}\right)} .
$$

So

$$
\left\|G_{m}(f)\right\|_{L^{2}\left(\boldsymbol{R}^{n}\right)} \leq C(\varphi) A\|f\|_{L^{2}\left(\boldsymbol{R}^{n}\right)},
$$

which combining the Littlewood-Paley theory, (3.13), (3.15), (3.16) with the proof of [4, p. 544, Lemma] leads to

$$
\left\|V_{j}^{m}(f)\right\|_{L^{p}\left(\boldsymbol{R}^{n}\right)} \leq C_{p} A\|f\|_{L^{p}\left(\boldsymbol{R}^{n}\right)}
$$

for $1 / 4=|1 / p-1 / 2|$. By interpolation between (3.20) and (3.21), we get from (3.18) that

$$
\left\|V_{\varepsilon}^{m}(f)\right\|_{L^{p}\left(\boldsymbol{R}^{n}\right)} \leq C(\varphi) A\|f\|_{L^{p}\left(\boldsymbol{R}^{n}\right)} \text { for } p \in(4 / 3,4) \text {. }
$$

Then

$$
\left\|G_{m}(f)\right\|_{L^{p}\left(\boldsymbol{R}^{n}\right)} \leq C(\varphi) A\|f\|_{L^{p}\left(\boldsymbol{R}^{n}\right)} \text { for } p \in(4 / 3,4)
$$


Reasoning as above, (3.13) together with (3.15), (3.16), (3.22), the proof of [4, p. 544, Lemma], the Littlewood-Paley theory and interpolation implies

$$
\left\|V_{\varepsilon}^{m}(f)\right\|_{L^{p}\left(\boldsymbol{R}^{n}\right)} \leq C(\varphi) A\|f\|_{L^{p}\left(\boldsymbol{R}^{n}\right)} \text { for } p \in(8 / 7,8) .
$$

By using this argument repeatedly, we can obtain ultimately that

$$
\left\|V_{\varepsilon}^{m}(f)\right\|_{L^{p}\left(\boldsymbol{R}^{n}\right)} \leq C(\varphi) A\|f\|_{L^{p}\left(\boldsymbol{R}^{n}\right)} \text { for } p \in(1, \infty) .
$$

This completes the proof of Lemma 3.3.

Now we return to the proof of Lemma 3.2. By Lemma 3.3, we have (3.17). This proves (3.12) for $\lambda=m$. Therefore we have (3.12) for all $\lambda \in\{0,1, \ldots, N\}$. Lemma 3.2 is proved.

By Lemma 3.2 and (3.11), we obtain the following lemma.

LEMMA 3.4. Let $\varphi \in \mathfrak{F}_{1}$ or $\mathfrak{F}_{2}$. For $\lambda \in\{1, \ldots, N\}$ and $i=1,2$, the operator $\mu_{\lambda}^{*, i}$ satisfies

$$
\left\|\mu_{\lambda}^{*, i}(f)\right\|_{L^{p}\left(\boldsymbol{R}^{n}\right)} \leq C_{p} A\|f\|_{L^{p}\left(\boldsymbol{R}^{n}\right)}
$$

for $1<p<\infty$. The constant $C_{p}$ is independent of $q, \gamma$ and the coefficients of $P_{\lambda}$, but depends on $\varphi$.

By combining the proof of $[4$, p. 544, Lemma] with (3.9) and (3.10), this lemma implies the following result.

LeMmA 3.5. Let $\varphi \in \mathfrak{F}_{1}$ or $\mathfrak{F}_{2}$. For $\lambda \in\{1, \ldots, N\}$ and $i=1,2$, the vector valued inequality

$$
\left\|\left(\sum_{k \in \boldsymbol{Z}}\left|\mu_{k, \lambda}^{i} * g_{k}\right|^{2}\right)^{1 / 2}\right\|_{L^{p}\left(\boldsymbol{R}^{n}\right)} \leq C_{p} A\left\|\left(\sum_{k \in \boldsymbol{Z}}\left|g_{k}\right|^{2}\right)^{1 / 2}\right\|_{L^{p}\left(\boldsymbol{R}^{n}\right)}
$$

holds for $1<p<\infty$. The constant $C_{p}$ is independent of $q, \gamma$ and the coefficients of $P_{\lambda}$, but depends on $\varphi$.

Proof OF THEOREM 1.2. We only prove the case $\varphi \in \mathfrak{F}_{1}$, since the other case can be obtained similarly. Let the operator $S_{j}$ be as in the proof of Lemma 3.3. It follows from (3.1) and (3.8) that

$$
\begin{aligned}
T_{h, \Gamma}(f)(\xi) & =\sum_{k \in \boldsymbol{Z}} \sigma_{k, N} * f(\xi) \\
& =\sum_{\lambda=1}^{N} \sum_{k \in \boldsymbol{Z}} \mu_{k, \lambda}^{1} * f(\xi) \\
& :=\sum_{\lambda=1}^{N} T_{\lambda}(f)(\xi) .
\end{aligned}
$$

So it suffices to obtain that

$$
\left\|T_{\lambda}(f)\right\|_{L^{p}\left(\boldsymbol{R}^{n}\right)} \leq C_{p} A\|f\|_{L^{p}\left(\boldsymbol{R}^{n}\right)}
$$


for $1<p<\infty$ and $\lambda \in\{1,2, \ldots, N\}$, where the constant $C_{p}$ is independent of $q, \gamma$ and the coefficients of $P_{\lambda}$, but depends on $\varphi$. We can write

$$
\begin{aligned}
T_{\lambda}(f)(\xi) & =\sum_{k \in \boldsymbol{Z}} \mu_{k, \lambda}^{1} * f(\xi) \\
& =\sum_{k \in \boldsymbol{Z}} \mu_{k, \lambda}^{1} * \sum_{j \in \boldsymbol{Z}} S_{j+k} S_{j+k} f(\xi) \\
& =\sum_{j \in \boldsymbol{Z}} \sum_{k \in \boldsymbol{Z}} S_{j+k}\left(\mu_{k, \lambda}^{1} * S_{j+k} f\right)(\xi) \\
& :=\sum_{j \in \boldsymbol{Z}} T_{j}(f)(\xi) .
\end{aligned}
$$

By (3.9) and the same argument as in getting (3.20), we have

$$
\left\|T_{j}(f)\right\|_{L^{2}\left(\boldsymbol{R}^{n}\right)} \leq C A\left(B_{\varphi}^{-j+2} \chi_{\{j \geq 2\}}+B_{\varphi}^{j+1} \chi_{\{j<2\}}\right)\|f\|_{L^{2}\left(\boldsymbol{R}^{n}\right)} .
$$

On the other hand, by the Littlewood-Paley theory and Lemma 3.5, we have

$$
\left\|T_{j}(f)\right\|_{L^{p}\left(\boldsymbol{R}^{n}\right)} \leq C A\|f\|_{L^{p}\left(\boldsymbol{R}^{n}\right)}
$$

for $1<p<\infty$. (3.25) and interpolation between (3.26) and (3.27) imply (3.24). Theorem 1.2 is finished.

Proof of Theorem 1.3. We only prove in the case $\varphi \in \mathfrak{F}_{1}$. The other case can be obtained by a similar argument. We shall use the method in [7]. For any $\varepsilon>0$, there exists an integer $k$ such that $\beta^{k-1} \leq \varepsilon<\beta^{k}$. Then by (3.8)

$$
\begin{aligned}
T_{h, \Gamma}^{*}(f)(\xi) & \leq \sigma_{N}^{*}(|f|)(\xi)+\sup _{k \in \boldsymbol{Z}}\left|\sum_{j=k}^{\infty} \sigma_{j, N} * f(\xi)\right| \\
& \leq \sigma_{N}^{*}(|f|)(\xi)+\sum_{\lambda=1}^{N} \sup _{k \in \boldsymbol{Z}}\left|\sum_{j=k}^{\infty} \mu_{j, \lambda}^{1} * f(\xi)\right| .
\end{aligned}
$$

By Lemma 3.2, it suffices to obtain that

$$
\left\|\sup _{k \in \boldsymbol{Z}}\left|\sum_{j=k}^{\infty} \mu_{j, \lambda}^{1} * f(\xi)\right|\right\|_{L^{p}\left(\boldsymbol{R}^{n}\right)} \leq C_{p} A\|f\|_{L^{p}\left(\boldsymbol{R}^{n}\right)}
$$

for $1<p<\infty$ and $\lambda \in\{1,2, \ldots, N\}$, where the constant $C_{p}$ is independent of $q, \gamma$ and the coefficients of $P_{\lambda}$, but depends on $\varphi$. Take $\beta=2^{q^{\prime} \gamma^{\prime}}$ and choose a radial function $\phi \in \mathscr{S}(\boldsymbol{R})$ (the Schwartz class) such that $\phi(\xi) \equiv 1$ when $|\xi|<1$ and $\phi(\xi) \equiv 0$ when $|\xi|>B_{\varphi}$. Let $\widehat{\Phi_{k}}(\xi)=\phi\left(\varphi\left(2^{q^{\prime} \gamma^{\prime} k}\right)^{\lambda}\left|a_{\lambda} \xi\right|\right)$, then

$$
\begin{aligned}
& \sum_{j=k}^{\infty} \mu_{j, \lambda}^{1} * f(\xi)=\left(\delta-\Phi_{k}\right) * \sum_{j=k}^{\infty} \mu_{j, \lambda}^{1} * f(\xi)+\Phi_{k} * T_{\lambda}(f)(\xi)-\Phi_{k} * \sum_{j=-\infty}^{k-1} \mu_{j, \lambda}^{1} * f(\xi) \\
&:=I_{k, 1}(f)(\xi)+I_{k, 2}(f)(\xi)+I_{k, 3}(f)(\xi),
\end{aligned}
$$


where $\delta$ is the Dirac delta function and $T_{\lambda}$ is as in the proof of Theorem 1.2. So

(3.30) $\sup _{k \in \boldsymbol{Z}}\left|\sum_{j=k}^{\infty} \mu_{j, \lambda}^{1} * f(\xi)\right| \leq \sup _{k \in \boldsymbol{Z}}\left|I_{k, 1}(f)(\xi)\right|+\sup _{k \in \boldsymbol{Z}}\left|I_{k, 2}(f)(\xi)\right|+\sup _{k \in \boldsymbol{Z}}\left|I_{k, 3}(f)(\xi)\right|$.

We obtain from (3.24) that

$$
\left\|\sup _{k \in \boldsymbol{Z}}\left|I_{k, 2}(f)\right|\right\|_{L^{p}\left(\boldsymbol{R}^{n}\right)} \leq C\left\|\mathcal{M}\left(T_{\lambda}(f)\right)\right\|_{L^{p}\left(\boldsymbol{R}^{n}\right)} \leq C_{p} A\|f\|_{L^{p}\left(\boldsymbol{R}^{n}\right)}
$$

for all $1<p<\infty$. Next, we estimate $\sup _{k \in \boldsymbol{Z}}\left|I_{k, 1}(f)\right|$. It holds that

$$
\sup _{k \in \boldsymbol{Z}}\left|I_{k, 1}(f)(\xi)\right| \leq \sum_{j=0}^{\infty} \sup _{k \in \boldsymbol{Z}}\left|\left(\delta-\Phi_{k}\right) * \mu_{j+k, \lambda}^{1} * f(\xi)\right|:=\sum_{j=0}^{\infty} \Lambda_{j}(f)(\xi) .
$$

By Lemma 3.4, we have

$$
\left\|\Lambda_{j}(f)\right\|_{L^{p}\left(\boldsymbol{R}^{n}\right)} \leq C_{p}\left\|\mu_{\lambda}^{*, 1}(|f|)\right\|_{L^{p}\left(\boldsymbol{R}^{n}\right)} \leq C_{p} A\|f\|_{L^{p}\left(\boldsymbol{R}^{n}\right)} \text { for } 1<p<\infty .
$$

On the other hand, by the Plancherel theorem and the choice of $\Phi_{k}$, we have

$$
\begin{aligned}
\left\|\Lambda_{j}(f)\right\|_{L^{2}\left(\boldsymbol{R}^{n}\right)}^{2} & \leq\left\|\left(\left|\left(\delta-\Phi_{k}\right) * \mu_{j+k, \lambda}^{1} * f\right|^{2}\right)^{1 / 2}\right\|_{L^{2}\left(\boldsymbol{R}^{n}\right)}^{2} \\
& \leq \sum_{k \in \boldsymbol{Z}} \int_{\left\{\varphi\left(2^{q^{\prime} \gamma^{\prime} k}\right)^{\lambda}\left|a_{\lambda} \xi\right| \geq 1\right\}}\left|\widehat{\mu_{j+k, \lambda}^{1}}(\xi)\right|^{2}|\hat{f}(\xi)|^{2} d \xi \\
& \leq \sum_{k \in \boldsymbol{Z}} \sum_{i=-\infty}^{k} \int_{\left\{\varphi\left(2^{q^{\prime} \gamma^{\prime} i}\right)^{-\lambda} \leq\left|a_{\lambda} \xi\right|<\varphi\left(2^{\left.\left.q^{\prime} \gamma^{\prime}(i-1)\right)-\lambda\right\}}\right.\right.}\left|\widehat{\mu_{j+k, \lambda}^{1}}(\xi)\right|^{2}|\hat{f}(\xi)|^{2} d \xi \\
& \leq C \sum_{k \in \boldsymbol{Z}} \sum_{i=-\infty}^{k}\left(A B_{\varphi}^{-j-k+i}\right)^{2} \int_{\left\{\varphi\left(2^{\prime} \gamma^{\prime} i\right)^{-\lambda} \leq\left|a_{\lambda} \xi\right|<\varphi\left(2 q^{\prime} \gamma^{\prime}(i-1)\right)^{-\lambda}\right\}}|\hat{f}(\xi)|^{2} d \xi \\
& \leq C A^{2} B_{\varphi}^{-2 j} \sum_{i=0}^{\infty} B_{\varphi}^{-2 i}\|f\|_{L^{2}\left(\boldsymbol{R}^{n}\right)}^{2} \\
& \leq C(\varphi) A^{2} B_{\varphi}^{-2 j}\|f\|_{L^{2}\left(\boldsymbol{R}^{n}\right)}^{2} .
\end{aligned}
$$

Then

$$
\left\|\Lambda_{j}(f)\right\|_{L^{2}\left(\boldsymbol{R}^{n}\right)} \leq C(\varphi) A B_{\varphi}^{-j}\|f\|_{L^{2}\left(\boldsymbol{R}^{n}\right)} .
$$

It follows from (3.32) and interpolation between (3.33) and (3.34) that

$$
\left\|\sup _{k \in \boldsymbol{Z}}\left|I_{k, 1}(f)\right|\right\|_{L^{p}\left(\boldsymbol{R}^{n}\right)} \leq C(\varphi) A\|f\|_{L^{p}\left(\boldsymbol{R}^{n}\right)} .
$$


Finally, we estimate $\sup _{k \in \boldsymbol{Z}}\left|I_{k, 3}(f)\right|$.

$$
\begin{aligned}
\sup _{k \in \boldsymbol{Z}}\left|I_{k, 3}(f)(\xi)\right| & =\sup _{k \in \boldsymbol{Z}}\left|\sum_{j=1}^{\infty} \Phi_{k} * \mu_{k-j}^{1} * f(\xi)\right| \\
& \leq \sum_{j=1}^{\infty} \sup _{k \in \boldsymbol{Z}}\left|\Phi_{k} * \mu_{k-j}^{1} * f(\xi)\right| \\
& :=\sum_{j=1}^{\infty} H_{j}(f)(\xi) .
\end{aligned}
$$

By Lemma 3.4, we have

$$
\left\|H_{j}(f)\right\|_{L^{p}\left(\boldsymbol{R}^{n}\right)} \leq C_{p} A\|f\|_{L^{p}\left(\boldsymbol{R}^{n}\right)} \text { for } 1<p<\infty .
$$

On the other hand, one can easily check that

$$
H_{j}(f)(\xi) \leq\left(\sum_{k \in \boldsymbol{Z}}\left|\Phi_{k} * \mu_{k-j}^{1} * f(\xi)\right|^{2}\right)^{1 / 2} .
$$

Thus by the Plancherel theorem and (3.9), we obtain

$$
\begin{aligned}
& \left\|H_{j}(f)\right\|_{L^{2}\left(\boldsymbol{R}^{n}\right)}^{2} \\
& \leq\left\|\left(\sum_{k \in \boldsymbol{Z}}\left|\Phi_{k} * \mu_{k-j}^{1} * f\right|^{2}\right)^{1 / 2}\right\|_{L^{2}\left(\boldsymbol{R}^{n}\right)}^{2} \\
& \leq\left.\sum_{k \in Z} \int_{\left\{\varphi\left(2 q^{\prime} \gamma^{\prime} k\right)^{\lambda}\left|a_{\lambda} \xi\right| \leq B_{\varphi}\right\}} \widehat{\mid \mu_{k-j, \lambda}^{1}}(\xi)\right|^{2}|\hat{f}(\xi)|^{2} d \xi \\
& \leq C \int_{\boldsymbol{R}^{n}} \sum_{k \in \boldsymbol{Z}}\left|\widehat{\mu_{k-j, \lambda}^{1}}(\xi)\right|^{2} \chi_{\left\{\varphi\left(2 q^{\prime} \gamma^{\prime} k\right)^{\lambda}\left|a_{\lambda} \xi\right| \leq B_{\varphi}\right\}}|\hat{f}(\xi)|^{2} d \xi \\
& \leq C A^{2} \sup _{\xi \in \boldsymbol{R}^{n}} \sum_{k \in \boldsymbol{Z}}\left|\varphi\left(2^{q^{\prime} \gamma^{\prime}(k-j+1)}\right)^{\lambda} a_{\lambda} \xi\right|^{1 /\left(q^{\prime} \gamma^{\prime} \lambda\right)} \chi_{\left\{\varphi\left(2^{\prime} \gamma^{\prime} k\right)^{\lambda}\left|a_{\lambda} \xi\right| \leq B_{\varphi}\right\}}\|f\|_{L^{2}\left(\boldsymbol{R}^{n}\right)}^{2} \\
& \leq C A^{2} B_{\varphi}^{2(-j+1)} \sup _{\xi \in \boldsymbol{R}^{n}} \sum_{k \in \boldsymbol{Z}}\left|\varphi\left(2^{q^{\prime} \gamma^{\prime} k}\right)^{\lambda} a_{\lambda} \xi\right|^{2 /\left(q^{\prime} \gamma^{\prime} \lambda\right)} \chi_{\left\{\varphi\left(2^{q^{\prime} \gamma^{\prime} k}\right)^{\lambda}\left|a_{\lambda} \xi\right| \leq B_{\varphi}\right\}}\|f\|_{L^{2}\left(\boldsymbol{R}^{n}\right)}^{2} \\
& \leq C(\varphi) A^{2} B_{\varphi}^{2(-j+1)}\|f\|_{L^{2}\left(\boldsymbol{R}^{n}\right)}^{2},
\end{aligned}
$$

where the last inequality is obtained by the properties of lacunary sequence. So

$$
\left\|H_{j}(f)\right\|_{L^{2}\left(\boldsymbol{R}^{n}\right)} \leq C(\varphi) A B_{\varphi}^{-j+1}\|f\|_{L^{2}\left(\boldsymbol{R}^{n}\right)} .
$$

The interpolation between (3.37) and (3.38) combining with (3.36) yields

$$
\left\|\sup _{k \in \boldsymbol{Z}}\left|I_{k, 3}(f)\right|\right\|_{L^{p}\left(\boldsymbol{R}^{n}\right)} \leq C(\varphi) A\|f\|_{L^{p}\left(\boldsymbol{R}^{n}\right)} .
$$

(3.29) follows from (3.30), (3.31), (3.35) and (3.39). This proves Theorem 1.3.

Proof of THEOREM 1.4. One can easily obtain the proof of Theorem 1.4 by Theorems 1.2 and 1.3 and the same arguments as in [13, Theorem 1.4] and [14, Theorem 2]. The details are omitted. 


\section{REFERENCES}

[ 1 ] W. C. Connetr, Singular integral near $L^{1}$. Harmonic analysis in Euclidean space (Proc. Sympos. Pure Math., WilliamsColl., Williamstown, Mass., 1978), Part 1, pp. 163-165, Proc. Sympos. Pure Math. 35, Part, Amer. Math. Soc., Providence, R.I., 1979.

[ 2 ] A. P. Calderó N ANd A. Zygmund, On singular integral, Amer. J. Math. 78 (1956), 289-309.

[3] A. Al-Salman And Y. PAn, Singular integrals with rough kernels in $L \log ^{+} L\left(S^{n-1}\right)$, J. London Math. Soc. 66 (2002), 153-174.

[ 4 ] J. Duoandikoetxea And J. L. Rubio de Francia, Maximal and singular integral operators via Fourier transform estimates, Invent. Math. 84 (1986), 541-561.

[ 5 ] Y. Ding, Q. XUE And K. YabutA, Boundedness of the Marcinkiewicz integrals with rough kernel associated to surfaces, Tohoku Math. J. 62 (2010), 233-262.

[6] R. Fefferman, A note on singular integrals, Proc. Amer. Math. Soc. 74 (1979), 266-270.

[ 7 ] D. FAN, K. GUO AND Y. PAN, A note of a rough singular integral operator, Math. Inequal. Appl. 1 (1999), 73-81.

[ 8 ] D. FAN AND Y. PAN, Singular integral operators with rough kernels supported by subvarieties, Amer. J. Math. 119 (1997), 799-839.

[9] F. LIU AND H. WU, Multiple singular integrals and Marcinkiewicz integrals with mixed homogeneity along surfaces, J. Inequ. Appl. 2012 (2012), 1-23.

[10] J. NAmaZI, A singular integral, Proc. Amer. Math. Soc. 96 (1986), 201-219.

[11] R. RiCCI AND E. M. STEIN, Harmonic analysis on nilpotent groups and singular integrals I: Oscillatory integrals, J. Funct. Anal. 73 (1987), 179-184.

[12] F. Ricci And G. Weiss, A characterization of $H^{1}\left(\sum_{n-1}\right)$, Harmonic analysis in Euclidean space (Proc. Sympos. Pure Math., Williams Coll., Williamstown, Mass., 1978), Part 1, pp. 289-294, Proc. Sympos. Pure Math. 35, Part, Amer. Math. Soc., Providence, R.I., 1979.

[13] S. S ATO, Estimates for singular integrals along surfaces of revolution, J. Aust. Math. Soc. 86 (2009), $413-430$.

[14] S. S ATo, Estimates for singular integrals and extrapolation, Studia Math. 192 (2009), 219-233.

[15] E. M. Stein, Harmonic Analysis: Real-variable methods, orthogonality and oscillatory integral, Princeton Math. Ser. 43, Monographs in Harmonic Analysis, III. Princeton University Press, Princeton, NJ, 1993.

[16] S. YANO, An extrapolation theorem, J. Math. Soc. Japan. 3 (1951), 296-305.

SCHOOL OF MATHEMATICAL SCIENCES

XIAMEN UNIVERSITY

Xiamen, Fujian 361005

P. R. CHINA

E-mail address: liufeng860314@163.com 